\title{
Positional voting rules generated by aggregation functions and the role of duplication
}

\author{
José Luis García-Lapresta \\ PRESAD Research Group, BORDA Research Unit, IMUVA, Departamento de Economía \\ Aplicada, Universidad de Valladolid, Spain \\ Miguel Martínez-Panero \\ PRESAD Research Group, BORDA Research Unit, IMUVA, Departamento de Economía \\ Aplicada, Universidad de Valladolid, Spain
}

\begin{abstract}
In this paper, we consider a typical voting situation where a group of agents show their preferences over a set of alternatives. Under our approach, such preferences are codified into individual positional values which can be aggregated in several ways through particular functions, yielding positional voting rules and providing a social result in each case. We show that scoring rules belong to such class of positional voting rules. But if we focus our interest on OWA operators as aggregation functions, other well-known voting systems naturally appear. In particular, we determine those ones verifying duplication (i.e., clone irrelevance) and present a proposal of an overall social result provided by them.

Keywords: positional voting rules, scoring rules, aggregation functions, OWA operators, duplication.
\end{abstract}

\section{Introduction}

There exists in Social Choice a long tradition controversy between the positional and non-positional approaches to voting theory, coming early from

Email addresses: lapresta@eco.uva.es (José Luis García-Lapresta), panero@eco.uva.es (Miguel Martínez-Panero)

Preprint submitted to International Journal of Intelligent Systems 
Borda and Condorcet, respectively. Gärdenfors [26] established a comprehensive framework to understand this opposition, and considered that "positionalist voting functions are those social choice functions where the positions of the alternatives in the voters' preference orders crucially influence the social ordering of the alternatives". Of course, this assertion can be understood in different ways $^{1}$, being Borda, plurality and antiplurality rules the most popular cases of positional voting rules. All of them are specific cases of scoring rules ${ }^{2}$, where the alternatives are socially ordered taking into account the sum of individual scores according to the agents' preferences. In fact, from Riker [41] on, many authors have identified scoring rules with positional voting methods. However, as we will show, scoring rules are not exclusive in capturing positional features of voting.

Our proposal is based on aggregation functions, mainly through $O W A$ operators $^{3}$. This tool has been revealed as a unifying way to face different issues appearing in several fields, Social Choice Theory among them (on this particular matter, see Wang et al. [46], Llamazares [35], García-Lapresta et al. [21] and Kacprzyk et al. [30], among others). As will be shown along the paper, this approach sheds light on some aspects avoided in the scoring context ${ }^{4}$.

One of these interesting properties, not satisfied by the scoring rules, is duplication. This property entails irrelevance of clone voters in the final outcome and might not seem suitable at all in voting scenarios. Nonetheless, its fulfillment should be convenient in several contexts; for example, when multiple votes are allowed for each voter (what happens if some Internet mechanisms are used) or whenever that streams of opinion, rather than individual opinions, should be

\footnotetext{
${ }^{1}$ See Pattanaik [39], especially Section 3.

${ }^{2}$ See Chebotarev and Shamis [12] for a referenced survey on scoring rules and their characterizations.

${ }^{3}$ The initials in OWA stand for ordered weighted averaging (see Yager [47], Yager and Kacprzyk [49] and Yager et al. [50]). This kind of operators have been characterized by Fodor et al. [19].

${ }^{4}$ This argument, supported along the present paper, already preliminarily appeared in García-Lapresta and Martínez-Panero [24].
} 
taken into account. Even more, it will be established that duplication arises in some specific positional voting rules induced by OWA operators interestingly related to decision under complete ignorance.

It is worth pointing out that there exist in the literature other possible alternative approaches extending the framework of scoring rules, such as flexible scoring rules introduced by Baharad and Nitzan [1]. On their hand, Xia and Conitzer $[54,55]$ have proposed what they call generalized scoring rules, extending well known voting rules such as Copeland, maximin, Bucklin and, of course, scoring rules. And recently Llamazares and Peña [36, 37] have employed cumulative standing functions representing in an interesting comprehensive way scoring rules such as plurality, antiplurality, Borda rule, $k$-approval voting, etc. Even more, these last authors also include in their proposal other voting rules based on variable scoring vectors or taking into account the support behind the candidates.

The rest of the paper is organized as follows. In Section 2 we introduce the basic notation for the voters' preferences over the alternatives and their related positions. Section 3 is devoted to voting rules as aggregation functions; we show that scoring rules are specific cases of such positional rules, and then we focus our attention on OWA operators and show their connections with some well-known voting rules appearing in the literature. The need of taking into account a variable electorate leads us to use extended OWA operators (EOWA operators) and, with this background, in Section 4 we define duplication and then we characterize those OWA-generated positional voting rules satisfying this property. An illustrative example is also presented, and a proposal of an overall social order based on the characterized rules is obtained in a unifying way. Finally, some concluding remarks are included in Section 5.

\section{Preliminaries}

Consider a set of voters $V=\{1, \ldots, m\}$, with $m \geq 2$ (occasionally, just for completion reasons, we will also consider the trivial case $m=1$ ). These voters 
show their preferences on a set of alternatives $X=\left\{x_{1}, \ldots, x_{n}\right\}$, with $n \geq 2$. With $L(X)$ we denote the set of linear orders on $X$, and with $W(X)$ the set of weak orders (or complete preorders) on $X$. Given $R, \succcurlyeq \in W(X)$, with $\succ$ and $\sim$ we denote the asymmetric and the symmetric parts of $R$, respectively. A profile is a vector $\boldsymbol{R}=\left(R_{1}, \ldots, R_{m}\right)$ of weak orders, where $R_{v}$ represents the preferences of the voter $v \in V$. Vectors in $\mathbb{R}^{n}$ are denoted as $\boldsymbol{a}=\left(a_{1}, \ldots, a_{n}\right)$. Given $\boldsymbol{a}, \boldsymbol{b} \in \mathbb{R}^{n}$, with $\boldsymbol{a} \leq \boldsymbol{b}$ we mean $a_{i} \leq b_{i}$ for every $i \in\{1, \ldots, n\}$.

Definition 1. Given $R \in W(X)$, the position of alternative $x_{i} \in X$ is defined as

$$
p\left(x_{i}\right)=n-\#\left\{x_{j} \in X \mid x_{i} \succ x_{j}\right\}-\frac{1}{2} \#\left\{x_{j} \in\left(X \backslash\left\{x_{i}\right\}\right) \mid x_{j} \sim x_{i}\right\} .
$$

It is worth mentioning that this proposal of assessing positions to the alternatives is equivalent to the way of scoring in extended versions of the Borda count for weak orders, where the scores of the tied alternatives are obtained as the average of the corresponding ones after a linearization process (see Smith [42], Black [8] and Cook and Seiford [14]).

Example 1. Consider $R \in W\left(\left\{x_{1}, \ldots, x_{7}\right\}\right)$ given by

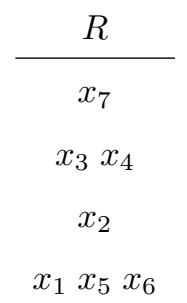

Then,

$$
\begin{aligned}
& p\left(x_{1}\right)=p\left(x_{5}\right)=p\left(x_{6}\right)=\frac{5+6+7}{3}=6=7-0-\frac{1}{2} 2, \\
& p\left(x_{2}\right)=4=7-3-\frac{1}{2} 0 \\
& p\left(x_{3}\right)=p\left(x_{4}\right)=\frac{2+3}{2}=2.5=7-4-\frac{1}{2} 1, \\
& p\left(x_{7}\right)=1=7-6-\frac{1}{2} 0 .
\end{aligned}
$$


Consequently, $R$ is codified by the positions vector

$$
\left(p\left(x_{1}\right), p\left(x_{2}\right), p\left(x_{3}\right), p\left(x_{4}\right), p\left(x_{5}\right), p\left(x_{6}\right), p\left(x_{7}\right)\right)=(6,4,2.5,2.5,6,6,1) .
$$

In the particular case of linear orders, positions for each alternative vary from 1 to $n$ with step 1 and the coordinates of each positions vector are permutations of $\mathcal{P}_{l}=\{1,2, \ldots, n\}$. In the general case of weak orders, it is easy to check that possible positions range from 1 to $n$ with step 0.5 , i.e., in this case the positional vectors take their coordinates in

$$
\mathcal{P}=\{1+\lambda \cdot 0.5 \mid \lambda \in\{0,1, \ldots, 2(n-1)\}\}=\{1,1.5,2, \ldots, n-0.5, n\},
$$

although not all $n$-dimensional vectors with these values as coordinates do represent a weak order ${ }^{5}$.

Taking into account the positions of the alternatives, every profile $\boldsymbol{R} \in$ $W(X)^{m}$ has associated a position matrix containing the positions of the alternatives for all the voters

$$
\left(\begin{array}{cccc}
p_{1}\left(x_{1}\right) & p_{1}\left(x_{2}\right) & \cdots & p_{1}\left(x_{n}\right) \\
p_{2}\left(x_{1}\right) & p_{2}\left(x_{2}\right) & \cdots & p_{2}\left(x_{n}\right) \\
\cdots & \cdots & \cdots & \cdots \\
p_{m}\left(x_{1}\right) & p_{m}\left(x_{2}\right) & \cdots & p_{m}\left(x_{n}\right)
\end{array}\right),
$$

where $p_{v}\left(x_{i}\right)$ is the position of $x_{i}$ for voter $v$. Thus, row $v$ contains the positions of the alternatives according to voter $v$, and column $i$ contains the positions of the alternative $x_{i}$.

\section{The aggregation process}

Definition 2. Given a domain $\mathcal{D} \subseteq W(X)^{m}$, a voting rule on $\mathcal{D}$ is a mapping $F: \mathcal{D} \longrightarrow W(X)$ that satisfies the following conditions:

\footnotetext{
${ }^{5}$ The set of of all admisible positions vectors in the previous sense has been characterized by García-Lapresta and Pérez-Román [25].
} 
1. Anonymity: For every permutation $\pi$ on $\{1, \ldots, m\}$ and every profile $\boldsymbol{R} \in \mathcal{D}$,

$$
F\left(R_{\pi(1)}, \ldots, R_{\pi(m)}\right)=F\left(R_{1}, \ldots, R_{m}\right) .
$$

2. Neutrality: For every permutation $\sigma$ on $\{1, \ldots, n\}$ and every profile $\boldsymbol{R} \in$ $\mathcal{D}$,

$$
F\left(R_{1}^{\sigma}, \ldots, R_{m}^{\sigma}\right)=\left(F\left(R_{1}, \ldots, R_{m}\right)\right)^{\sigma},
$$

where $R_{v}^{\sigma}$ and $\left(F\left(R_{1}, \ldots, R_{m}\right)\right)^{\sigma}$ are the orders obtained from $R_{v}$ and $F\left(R_{1}, \ldots, R_{m}\right)$, respectively, by relabeling the alternatives according to $\sigma$, i.e., $x_{\sigma(i)} R_{v}^{\sigma} x_{\sigma(j)} \Leftrightarrow x_{i} R_{v} x_{j}$ and $x_{\sigma(i)}\left(F\left(R_{1}, \ldots, R_{m}\right)\right)^{\sigma} x_{\sigma(j)} \Leftrightarrow$ $x_{i} F\left(R_{1}, \ldots, R_{m}\right) x_{j}$.

3. Unanimity: For every profile $\boldsymbol{R} \in \mathcal{D}$ and all $x_{i}, x_{j} \in X$,

$$
\left(\forall v \in V \quad x_{i} R_{v} x_{j}\right) \Rightarrow x_{i} F(\boldsymbol{R}) x_{j} .
$$

Anonymity means a symmetric consideration for the voters; neutrality means a symmetric consideration for the alternatives; and unanimity means that if all the individuals consider an alternative as good as another one, then the social preference coincides with the individual preferences on this issue.

The previous framework considering voting rules, where the outcome is a social order (as in Smith [42]), is not unique at all in Social Choice Theory. Other possible approaches can be taken into account, such as social choice correspondences, where the result is the (nonempty) subset of the best alternatives (as in Young [52, 53]; see also Laslier [31] for further rank-based and pairwisebased approaches), or even social choice functions, where a single alternative is assigned to each profile ${ }^{6}$.

\subsection{Aggregation functions}

In our proposal, we have adapted the notion of aggregation function from $[0,1]^{m}$ to $m$-tuples of $[1, \infty)^{m}$. In fact, for our purposes, it suffices to deal

\footnotetext{
${ }^{6}$ As pointed out by Courtin et al. [15], differences in the axiomatic treatment arise depending on the type of social mechanism considered.
} 
with more restricted domains, namely, $\mathcal{P}^{m}=\{1,1.5,2, \ldots, n-0.5, n\}^{m}$ or $\mathcal{P}_{l}^{m}=\{1,2, \ldots, n\}^{m}$. On aggregation functions in the standard unit interval, see Calvo et al. [10], Beliakov et al. [7,6] and Grabisch et al. [27].

Definition 3. Let $D$ be a domain being $D=\mathcal{P}^{m}$ or $D=\mathcal{P}_{l}^{m}$. An aggregation function on $D$ is a mapping $A: D \longrightarrow \mathbb{R}$ verifying the following conditions:

1. Boundary conditions: $A(1, \ldots, 1)=1$ and $A(n, \ldots, n)=n$.

2. Monotonicity: $\boldsymbol{a} \leq \boldsymbol{b} \Rightarrow A(\boldsymbol{a}) \leq A(\boldsymbol{b})$, for all $\boldsymbol{a}, \boldsymbol{b} \in D$.

If, additionally, $A$ satisfies idempotency, i.e., $A(a, \ldots, a)=a$ for every $a \in \mathcal{P}$ (resp. $a \in \mathcal{P}_{l}$ ), then $A$ is called averaging aggregation function.

It is easy to see that averaging aggregation functions satisfy compensativeness:

$$
\min \left\{a_{1}, \ldots, a_{m}\right\} \leq A\left(a_{1}, \ldots, a_{m}\right) \leq \max \left\{a_{1}, \ldots, a_{m}\right\}
$$

for every $\left(a_{1}, \ldots, a_{m}\right) \in D$.

Typical averaging aggregation functions are the arithmetic mean, trimmed means, the median, the maximum, the minimum, etc. In fact, we can gather all these aggregation functions as specific cases of OWA operators.

Definition 4. $A$ weighting vector of dimension $m$ is a vector $\boldsymbol{w}=\left(w_{1}, \ldots, w_{m}\right) \in$ $[0,1]^{m}$ such that $\sum_{i=1}^{m} w_{i}=1$.

Definition 5. Given $D=\mathcal{P}^{m}$ or $D=\mathcal{P}_{l}^{m}$ and a weighting vector $\boldsymbol{w}$ of dimension $m$, the OWA operator on $D$ associated with $\boldsymbol{w}$ is the mapping $A_{\boldsymbol{w}}$ : $D \longrightarrow \mathbb{R}$ defined as

$$
A_{\boldsymbol{w}}\left(a_{1}, \ldots, a_{m}\right)=\sum_{i=1}^{m} w_{i} \cdot a_{[i]},
$$

where $a_{[i]}$ is the $i$-th greatest number of $a_{1}, \ldots, a_{m}$.

As noted before, some well-known aggregation functions are specific cases of OWA operators.

With appropriate weighting vectors $\boldsymbol{w}=\left(w_{1}, \ldots, w_{m}\right)$ we obtain 
1. The maximum, for $\boldsymbol{w}=(1,0, \ldots, 0)$.

2. The minimum, for $\boldsymbol{w}=(0, \ldots, 0,1)$.

3. The arithmetic mean, for $\boldsymbol{w}=\left(\frac{1}{m}, \ldots, \frac{1}{m}\right)$.

4. The k-trimmed means:

- If $k=1, \boldsymbol{w}=\left(0, \frac{1}{m-2}, \ldots, \frac{1}{m-2}, 0\right)$.

- If $k=2, \boldsymbol{w}=\left(0,0, \frac{1}{m-4}, \ldots, \frac{1}{m-4}, 0,0\right)$.

- ....

5. The median:

(a) If $m$ is odd, $w_{i}= \begin{cases}1, & \text { if } i=\frac{m+1}{2}, \\ 0, & \text { otherwise. }\end{cases}$

(b) If $m$ is even, $w_{i}= \begin{cases}\frac{1}{2}, & \text { if } i \in\left\{\frac{m}{2}, \frac{m}{2}+1\right\}, \\ 0, & \text { otherwise. }\end{cases}$

6. The mid-range, for $\boldsymbol{w}=(0.5,0, \ldots, 0,0.5)$.

For $m=3$, the set of weighting vectors

$$
\{(\alpha, \beta, 1-\alpha-\beta) \mid 0 \leq \alpha \leq 1,0 \leq \beta \leq 1, \alpha+\beta \leq 1\}
$$

can be identified with the triangle $\left\{(\alpha, \beta) \in[0,1]^{2} \mid \alpha+\beta \leq 1\right\}$ (see Figure 1).

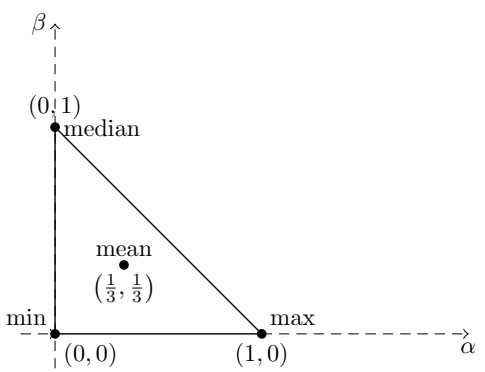

Figure 1: The main OWA operators for $m=3$.

Note that the vertices of the triangle, $(0,0),(1,0)$ and $(0,1)$, correspond to the minimum, the maximum and the median, respectively; and the baricenter of the triangle, $\left(\frac{1}{3}, \frac{1}{3}\right)$, corresponds to the arithmetic mean. 


\subsection{Positional voting rules}

Definition 6. Given the aggregation function $A: \mathcal{P}^{m} \longrightarrow \mathbb{R}$ and a profile $\boldsymbol{R} \in W(X)^{m}$, the aggregated position of the alternative $x_{i} \in X$ is defined as

$$
p_{A}\left(x_{i}\right)=A\left(p_{1}\left(x_{i}\right), \ldots, p_{m}\left(x_{i}\right)\right),
$$

where $p_{v}\left(x_{i}\right)$ is the position of $x_{i}$ for voter $v \in V$.

For every aggregation function $A: \mathcal{P}^{m} \longrightarrow \mathbb{R}$, we consider the mapping $F_{A}: W(X)^{m} \longrightarrow W(X)$ defined as $F_{A}(\boldsymbol{R})=\succcurlyeq_{A}$, where

$$
x_{i} \succcurlyeq_{A} x_{j} \Leftrightarrow p_{A}\left(x_{i}\right) \leq p_{A}\left(x_{j}\right)
$$

Remark 1. In the previous situation, it is easy to check that $F_{A}$ is a voting rule.

Definition 7. For every aggregation function $A: \mathcal{P}^{m} \longrightarrow \mathbb{R}, F_{A}$ is the positional voting rule associated with $A$.

\subsubsection{Scoring rules as positional voting rules}

As pointed out before, scoring rules appear to be the paradigm of the positional approach to voting theory. In what follows we define this class of rules, which encloses well-known voting rules such as plurality, antiplurality and Borda rules, among others. Then, we will show that all of them are positional voting rules in the aforementioned sense, but it will be shown that the reverse is not true.

Definition 8. A scoring vector of dimension $n \in \mathbb{N}$ is a vector $s=\left(s_{1}, \ldots, s_{n}\right) \in$ $\mathbb{R}^{n}$ such that $s_{1} \geq \cdots \geq s_{n}$ and $s_{1}>s_{n}$.

Now, suppose that voters' preferences over the alternatives are linear orders (i.e., weak orders where ties among distinct alternatives are avoided), gathered in a profile $\boldsymbol{R} \in L(X)^{m}$. Given a scoring vector $s=\left(s_{1}, \ldots, s_{n}\right)$, for each voter, $s_{1}$ points are assigned to the top-ranked alternative, $s_{2}$ points to the secondranked alternative, and so on. Formally, in terms of positions, the individual 
score of voter $v \in V$ for the alternative $x_{i}$ is $r_{\boldsymbol{s}}^{v}\left(x_{i}\right)=s_{p_{v}\left(x_{i}\right)}$. The collective score for the alternative $x_{i}$ is $r_{\boldsymbol{s}}\left(x_{i}\right)=\sum_{v=1}^{m} r_{\boldsymbol{s}}^{v}\left(x_{i}\right)$. The alternative(s) with the largest total score is (are) the winner(s).

Definition 9. Given a scoring vector $s$ of dimension n, the scoring rule associated with $s$ is the mapping $F_{\boldsymbol{s}}: L(X)^{m} \longrightarrow W(X)$ defined as $F_{\boldsymbol{s}}(\boldsymbol{R})=\succcurlyeq_{\boldsymbol{s}}$, where $x_{i} \succcurlyeq_{s} x_{j} \Leftrightarrow r_{\boldsymbol{s}}\left(x_{i}\right) \geq r_{\boldsymbol{s}}\left(x_{j}\right)$.

Remark 2. It is straightforward to check that $F_{\boldsymbol{s}}$ is a voting rule for every scoring vector $s$. In what follows, notice that when dealing with positional voting rules, the smallest value(s) correspond(s) to the best position(s), just the opposite happening with scoring rules, where the highest score determines the winner(s).

The following cases give the scoring rules associated with various voting rules appearing in the literature.

- $k$-approval voting ${ }^{7}, k \in\{1,2, \ldots, n-1\}: s=(1, \ldots, 1,0, \ldots, 0)$, with $k$ 1 's. As important specific cases of this, we have

- Plurality: for $k=1, s=(1,0, \ldots, 0)$.

- Antiplurality: for $k=n-1, s=(1, \ldots, 1,0)$.

- Borda rule: $s=(n-1, n-2, \ldots, 1,0)$.

- Best-worst voting rules ${ }^{8}: s=(1, s, \ldots, s, 0)$, with $s \in(0,1)$.

Note that the excluded cases $s=0$ and $s=1$ would correspond again to plurality and antiplurality, respectively.

\footnotetext{
${ }^{7}$ Notice that while $k$-approval voting is a scoring rule, approval voting (where each voter can approve of as many alternatives as wished) is not. However, it can be understood as a flexible scoring rule following the extended framework proposed by Baharad and Nitzan [1].

${ }^{8}$ Best-worst voting rules were introduced and axiomatically characterized in the scoring context by García-Lapresta et al. [22].
} 
Given the scoring rule associated with the scoring vector $\left(s_{1}, \ldots, s_{n}\right), a, b \in$ $\mathbb{R}$ such that $a>0$, the new scoring rule associated with the scoring vector $\left(s_{1}^{\prime}, \ldots, s_{n}^{\prime}\right)$, where $s_{i}^{\prime}=a s_{i}+b$ for every $i \in\{1, \ldots, n\}$, is equivalent to the previous one, in the sense that they provide the same social outcomes. In this way, every scoring vector $\left(s_{1}, \ldots, s_{n}\right)$ can be normalized, i.e., it is equivalent to $\left(s_{1}^{\prime}, \ldots, s_{n}^{\prime}\right)$ with $s_{1}^{\prime}=1$ and $s_{n}^{\prime}=0$, by simply taking $s_{i}^{\prime}=\left(s_{i}-s_{n}\right) /\left(s_{1}-s_{n}\right)$.

For $n=4$, the set of normalized scoring vectors $\{(1, s, t, 0) \mid 0 \leq t \leq s \leq 1\}$ can be identified with the triangle $\left\{(s, t) \in[0,1]^{2} \mid t \leq s\right\}$ (see García-Lapresta et al. [20]). As shown in Figure 2, the vertices of the triangle, $(0,0),(1,0)$ and $(1,1)$, correspond to plurality, 2-approval voting and antiplurality, respectively; the baricenter of the triangle, $\left(\frac{2}{3}, \frac{1}{3}\right)$, corresponds to the Borda rule; and the segment conecting $(0,0)$ and $(1,1)$ corresponds to the set of best-worst voting rules.

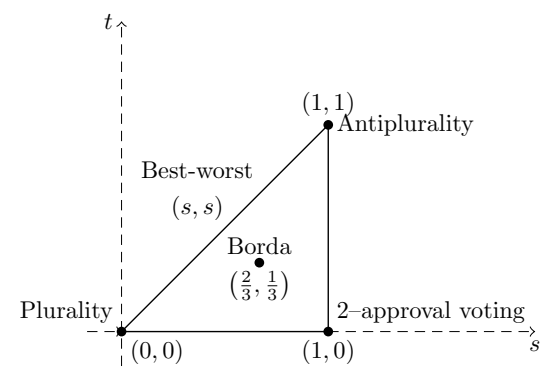

Figure 2: The best-known scoring rules for $n=4$.

The next result shows how to construct an aggregation function from any scoring vector so that their associated positional voting rule and the corresponding scoring rule are the same.

Proposition 1. All the scoring rules are positional voting rules.

PRoOF: Let $s=\left(s_{1}, \ldots, s_{n}\right) \in[0,1]^{n}$ be a normalized scoring vector, i.e., $1=s_{1} \geq s_{2} \geq \cdots \geq s_{n-1} \geq s_{n}=0$. Let $A_{s}: \mathcal{P}_{l}^{m} \longrightarrow \mathbb{R}$ be the mapping defined as

$$
A_{\boldsymbol{s}}\left(a_{1}, \ldots, a_{m}\right)=1+\frac{n-1}{m} \sum_{v=1}^{m} \varphi_{\boldsymbol{s}}\left(a_{v}\right)
$$


with $\varphi_{\boldsymbol{s}}(i)=1-s_{i}$ for every $i \in\{1, \ldots, n\}$. It is easy to see that $A_{\boldsymbol{s}}$ is an aggregation function on $\mathcal{P}_{l}^{m}$.

We also have

$$
\begin{aligned}
& x_{i} \succcurlyeq_{A_{\boldsymbol{s}}} x_{j} \Leftrightarrow p_{A_{\boldsymbol{s}}}\left(x_{i}\right) \leq p_{A_{\boldsymbol{s}}}\left(x_{j}\right) \Leftrightarrow \\
& A_{\boldsymbol{s}}\left(p_{1}\left(x_{i}\right), \ldots, p_{m}\left(x_{i}\right)\right) \leq A_{\boldsymbol{s}}\left(p_{1}\left(x_{j}\right), \ldots, p_{m}\left(x_{j}\right)\right) \Leftrightarrow \\
& 1+\frac{n-1}{m} \sum_{v=1}^{m} \varphi_{\boldsymbol{s}}\left(p_{v}\left(x_{i}\right)\right) \leq 1+\frac{n-1}{m} \sum_{v=1}^{m} \varphi_{\boldsymbol{s}}\left(p_{v}\left(x_{j}\right)\right) \Leftrightarrow \\
& r_{\boldsymbol{s}}\left(x_{i}\right)=\sum_{v=1}^{m} r_{\boldsymbol{s}}^{v}\left(x_{i}\right) \geq \sum_{v=1}^{m} r_{\boldsymbol{s}}^{v}\left(x_{j}\right)=r_{\boldsymbol{s}}\left(x_{j}\right) \Leftrightarrow x_{i} \succcurlyeq_{\boldsymbol{s}} x_{j} .
\end{aligned}
$$

Hence, $\succcurlyeq_{A s}=\succcurlyeq_{s}$ and, consequently, the scoring rule associated with $s$ coincides with the positional voting rule associated with $A_{\boldsymbol{s}}$.

Now we will show that our positional approach actually does extend the scoring context. We mean that, although sharing similar patterns, it is not true that every positional voting rule associated with an aggregation function can

\begin{tabular}{|c|c|c|}
\hline$R_{1}$ & $R_{2}$ & $R_{3}$ \\
\hline$x_{1}$ & $x_{1}$ & $x_{2}$ \\
\hline$x_{3}$ & $x_{3}$ & $x_{3}$ \\
\hline$x_{2}$ & $x_{2}$ & $x_{1}$ \\
\hline
\end{tabular}
be represented by a scoring rule (in the sense that both provide the same social order).

Example 2. Consider the profile given by

where the associated position matrix is

$$
\left(\begin{array}{lll}
1 & 3 & 2 \\
1 & 3 & 2 \\
3 & 1 & 2
\end{array}\right) .
$$

Aggregating through the maximum $(A=\max )$, i.e., under the maximin voting rule, the following social order is obtained: $x_{3} \succ_{A} x_{1} \sim_{A} x_{2}$. Let now consider 
a generic scoring rule with associated (normalized) scoring vector $\boldsymbol{s}=(1, s, 0)$, $0 \leq s \leq 1$. Then, the collective scores obtained for the alternatives are

$$
r_{\boldsymbol{s}}\left(x_{1}\right)=2, \quad r_{\boldsymbol{s}}\left(x_{2}\right)=1, \quad r_{\boldsymbol{s}}\left(x_{3}\right)=3 s .
$$

According to the results, every scoring rule provides $x_{1} \succ_{s} x_{2}$. In any case, none of them represents the positional voting rule associated with the maximum, where $x_{1} \sim_{A} x_{2}$, as pointed out before. Consequently, the class of positional voting rules does not coincide with the class of scoring rules.

Remark 3. Notice an interesting feature of the maximin rule that can be observed in the above situation: A kind of clone irrelevance, i.e., the influence of voter's copies is irrelevant in the final outcome. Thus, in Example 2, the presence or absence of voter 2 (a clone of voter 1 ) does not affect the result. This is a determinant fact for excluding the maximin voting rule from scoring rules, because it is against Young's [53] continuity $^{9}$, an axiom appearing in his characterization of scoring rules.

\subsubsection{OWA-generated positional voting rules}

Taking into account some of the OWA operators mentioned above, we obtain positional voting rules which are connected to (or even replicate) well-known procedures appearing in the literature:

- The arithmetic mean as aggregation function induces the Borda rule. And it is worth mentioning that the arithmetic mean is also the basis for the Range Voting method (Smith [43]), in a decisional context where the alternatives receive numerical assessments one by one.

- The median instead of the arithmetic mean, and linguistic terms instead of numerical values, are used in the Majority Judgment voting system in-

\footnotetext{
${ }^{9}$ This is an archimedean-type property which states that if two disjoint sets of voters $U$ and $V$ select $x$ and $y$ as winners, respectively, then $x$ should be a winner for the superset $(n U) \cup V$ for $n$ sufficiently large, where $(n U)$ means $n$ copies of those voters and their votes. Clearly, as exposed, this is not fulfilled by the maximin voting rule.
} 
troduced by Balinski and Laraki [3, 4]. Extensions of this procedure using centered OWA operators (Yager [48]) and distances appear in GarcíaLapresta and Martínez-Panero [23] and in Falcó and García-Lapresta [17], respectively. Again, in a different scenario, Bucklin's method selects the candidates with highest median ranking as winners (see Tideman [44] and Felsenthal [18]), and similarly Basset and Persky [5] also proposed to select the alternative with best median evaluation (see also Laslier [33], who has coined the term maxmed for this voting scheme).

- The maximum leads to a voting rule in which each alternative is evaluated according to the worst reached position. Those with the best assigned value are then elected. Such a maximin voting rule, which advocates the maximin principle of normative economics ${ }^{10}$, is called fallback bargaining ${ }^{11}$ by Brams and Kilgour [9]. It has been characterized in the voting context by Congar and Merlin [13] (see also Llamazares and Peña [36]).

The same underlying idea appears in the leximin voting system proposed by Laslier [32] (see also Laslier [33]), and in the Simpson-Kramer method (see Levin and Nalebuff [34]), although in different decisional frameworks. Furthermore, the procedure obtained through the maximum as aggregation operator is also related to the Coombs method (where the alternative with the largest number of last positions is sequentially withdrawn), as well as to the antiplurality rule (see Baharad and Nitzan [2] and Congar and Merlin [13]).

- The minimum entails a voting rule called $\operatorname{maximax}^{12}$ by Congar and

\footnotetext{
${ }^{10}$ Rawls [40, p. 328]: "the basic structure is perfectly just when the prospects of the least fortunate are as great as they can be".

${ }^{11}$ Concretely, the maximin rule corresponds to the case of fallback bargaining with unanimity, also called Kant-Rawls social compromise after Hurwicz and Sertel [29].

${ }^{12}$ The apparent discordance leading the maximum to the maximin voting rule, as well as the minimum to the maximax, relies on our positional approach where, contrary to the scoring context, the smallest value is associated with the best position, as pointed out before. It is
} 
Merlin [13], also characterized by them. Its conception is similar to that of the Hare rule, also known as alternative vote (where the alternative with the fewest first positions is sequentially withdrawn). It is also related to the most used (and criticized) voting system: plurality rule (see Laslier [32] and Congar and Merlin [13]).

- The mid-range is related to the basic 1-best-1-worst voting rule (see García-Lapresta et al. [22]).

Remark 4. There can exist a unique "translation" between weighting vectors and normalizated scoring vectors. This is what happens with the Borda rule: as a scoring rule, it is associated with $\left(1, \frac{n-2}{n-1}, \ldots, \frac{1}{n-1} 1,0\right)$, while as OWAgenerated positional voting rule corresponds to $\left(\frac{1}{m}, \ldots, \frac{1}{m}\right)$. However, in some cases such translation does not exist (see Remark 3, where it is shown that maximin rule can not be captured through any scoring rule; and it is also true for the maximax rule). Even more, depending on the situation, there can be several possibilities of translation from the same scoring rule into OWAgenerated positional voting rules, being not compatible among them. This fact is shown in what follows.

Consider the profile given by

$\begin{array}{cllll}\frac{R_{1}}{x_{1}} & & \frac{R_{2}}{x_{2}} & & \frac{R_{3}}{x_{3}} \\ x_{3} & & x_{3} & & x_{2} \\ x_{2} & & x_{1} & & x_{1}\end{array}$

The result under plurality rule is $x_{1} \sim x_{2} \sim x_{3}$, and it is easy to check that this social order is also the same under the OWA-generated positional voting rule associated with the weighting vector $(0,0,1)$, i.e., the maximax rule.

On the other hand, consider the new profile

also worth to note in what follows that in the scoring context there are as many scores as alternatives, whereas in the positional scenario, when dealing with OWA operators, there are as many weights as voters. 


\begin{tabular}{|c|c|c|}
\hline$R_{1}$ & $R_{2}$ & $R_{3}$ \\
\hline$x_{1}$ & $x_{1}$ & $x_{2}$ \\
\hline$x_{2}$ & $x_{3}$ & $x_{3}$ \\
\hline$x_{3}$ & $x_{2}$ & $x_{1}$ \\
\hline
\end{tabular}

Now plurality rule gives $x_{1} \succ x_{2} \succ x_{3}$ and, after some computation, the same social order can be obtained through every OWA-generated positional voting rule associated with any weighting vector $(\alpha, \beta, 1-\alpha-\beta)$ such that $\beta>0$.

Thus, these two profiles show that, even when existing weights associated with scores, they could have not common values. Concretely, in words referred to our example, being the first weighting vector incompatible with those obtained to capture plurality in the second profile, it can be argued that there not exists "the" OWA-generated positional voting rule associated with plurality (similar reasons for the antiplurality rule also stand).

Remark 5. One can ask if OWA-generated positional voting rules which are not scoring rules are those considering in the aggregation process only the information about the best and/or worst positioned alternatives, as happening with maximin and maximax rules. The answer is no. In both of the profiles appearing in Remark 4, the maxmed rule (whose weighting vector is $(0,1,0)$ ) cannot be represented under any scoring rule. For example, in the first profile, under a normalized scoring vector $(1, s, 0)$ with $s \in[0,1]$, the obtained social order is $x_{1} \sim x_{2} \sim x_{3}$ when $s=0$ (plurality, as aforementioned) and $x_{3} \succ x_{2} \succ x_{1}$ when $s>0$. However, if maxmed were applied, then the result would be $x_{3} \sim x_{2} \succ x_{1}$, incompatible with any of these scoring rules.

\subsection{Extended notions}

Sometimes it is necessary to take into account a variable electorate (for instance, as mentioned, to deal with the clonation or appearance of new voters, as happening in Example 2). This is the reason why we introduce some extended notions of those already defined throughout the paper. 
Definition 10. An extended voting rule is a mapping

$$
\widetilde{F}: \bigcup_{m \in \mathbb{N}} W(X)^{m} \longrightarrow W(X)
$$

such that $F_{m}=\left.\widetilde{F}\right|_{W(X)^{m}}$ is a voting rule for each dimension $m=2,3, \ldots$, and $F_{1}(R)=R$.

Definition 11. An extended OWA operator (EOWA) is a sequence of OWA operators $\widetilde{A}=\left(A_{\boldsymbol{w}^{m}}\right)_{m \in \mathbb{N}}$ with associated weighting vectors $\boldsymbol{w}^{m}=\left(w_{1}^{m}, \ldots, w_{m}^{m}\right)$, one for each dimension $m \in \mathbb{N}$.

Following Calvo and Mayor [11] and Mayor and Calvo [38] (see also Beliakov et al. [7, pp. 54-56]) and Beliakov et al. [6, pp. 73-76]), we can show graphically an EOWA operator as a weighting triangle where the entries in each row add up to one.

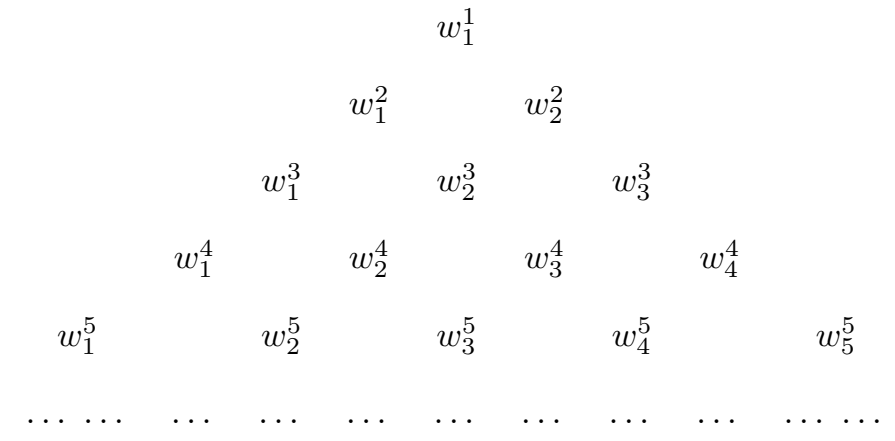

\section{Duplication}

Here we formally introduce the aforementioned clone irrelevance property which, broadly speaking, requires that new voters replicating the same preferences of already existing voters will not affect the social outcome. At first sight such statement might seem a vulneration of the very essence of democracy, but it can make sense in some contexts. For example, in bargaining, when the above mentioned fallback method is used to find a compromise among the bargainers 
because they "fall back in lockstep to less and less preferred positions until they agree on outcome" (see Brams and Kilgour [9]). But it also make sense in voting scenarios such as the Internet, where agents can cast their votes more than one time ${ }^{13}$; or wherever that, rather than merely the total amount of votes, different currents of opinion or electoral bodies (such as minorities) should be taken into account.

This property appears as duplication in Congar and Merlin [13], where they consider this axiom in order to capture situations of complete ignorance in some voting contexts (see references therein) and characterize the maximin procedure.

Definition 12. An extended voting rule $\widetilde{F}$ satisfies duplication if

$$
F_{m+1}\left(\boldsymbol{R}, R_{i}\right)=F_{m}(\boldsymbol{R})
$$

for every profile $\boldsymbol{R}=\left(R_{1}, \ldots, R_{i}, \ldots, R_{m}\right) \in W(X)^{m}$ and every $i \in\{1, \ldots, m\}$.

\subsection{A characterization result}

It is interesting to find those procedures satisfying duplication, and the following result shows the answer for positional voting rules associated with EOWA operators.

Theorem 1. Given an EOWA operator $\widetilde{A}=\left(A_{\boldsymbol{w}^{m}}\right)_{m \in \mathbb{N}}$, the extended voting rule $\widetilde{F}_{\widetilde{A}}$ satisfies duplication if and only if $\widetilde{A}$ is a rational convex combination of the maximum and the minimum EOWA operators, i.e., there exists $\alpha \in[0,1] \cap \mathbb{Q}$ such that $\boldsymbol{w}^{m}=\alpha(1,0, \ldots, 0)+(1-\alpha)(0, \ldots, 0,1)$ for every $m \in \mathbb{N}$.

Proof: It is straightforward that positional voting rules associated with $A_{\boldsymbol{w}^{m}}$, where $\boldsymbol{w}^{m}=(1,0, \ldots, 0)$ (i.e., maximin), $\boldsymbol{w}^{m}=(0,0, \ldots, 1)$ (i.e., $\operatorname{maximax}$ ),

\footnotetext{
${ }^{13}$ See Yokoo and Matsubara [51], where they analyze the effect of false name bids in Internet auctions as well as Wagman and Conitzer [45], where these authors deal with false-name-proof voting mechanisms, i.e., those where no agent benefits from participating more than once.
} 
and convex combinations of them, $\boldsymbol{w}^{m}=(\alpha, 0, \ldots, 0,1-\alpha)$, with $\alpha \in[0,1]$, satisfy duplication ${ }^{14}$.

For the reciprocal, we first prove that if duplication holds for an extended voting rule $\widetilde{F}_{\widetilde{A}}$, where $\widetilde{A}=\left(A_{\boldsymbol{w}^{m}}\right)_{m \in \mathbb{N}}$, then all intermediate weights in each dimension $m, w_{2}, \ldots, w_{m-1}$, should be zero. Our reasoning will deal with a profile consisting in all circular permutations ${ }^{15}$ of three alternatives, but the argument is extensible to $m>3$. Thus, consider the profile

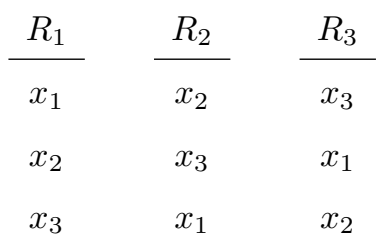

where the associated position matrix is

$$
\left(\begin{array}{lll}
1 & 2 & 3 \\
3 & 1 & 2 \\
2 & 3 & 1
\end{array}\right)
$$

As every alternative occupies each position exactly once, a global tie arises and the aggregated position for each is $p_{A}\left(x_{i}\right)=3 w_{1}^{3}+2 w_{2}^{3}+w_{3}^{3}, i=1,2,3$, so that $x_{1} \sim_{A_{\boldsymbol{w}^{3}}} x_{2} \sim_{A_{\boldsymbol{w}^{3}}} x_{3}$, where $\boldsymbol{w}^{3}=\left(w_{1}^{3}, w_{2}^{3}, w_{3}^{3}\right)$.

Now suppose that voter 1 is replicated, becoming the new situation

\begin{tabular}{|c|c|c|c|}
\hline$R_{1}$ & $R_{2}$ & $R_{3}$ & $R_{4}=R_{1}$ \\
\hline$x_{1}$ & $x_{2}$ & $x_{3}$ & $x_{1}$ \\
\hline$x_{2}$ & $x_{3}$ & $x_{1}$ & $x_{2}$ \\
\hline$x_{3}$ & $x_{1}$ & $x_{2}$ & $x_{3}$ \\
\hline
\end{tabular}

\footnotetext{
${ }^{14}$ Notice that, in the previous argument, $\alpha$ does not need to be rational. However, as pointed out by Fagin and Wimmers [16] "in some situations we have to restrict our attention to rational weights". One of these situations naturally arises when "we simply allow multiple copies of voters", which is exactly our case.

${ }^{15}$ These circular permutations yield a Condorcet cycle.
} 
where the new associated position matrix is

$$
\left(\begin{array}{lll}
1 & 2 & 3 \\
3 & 1 & 2 \\
2 & 3 & 1 \\
1 & 2 & 3
\end{array}\right) .
$$

Then, the aggregated positions for each alternative are

$$
\begin{aligned}
& p_{A}\left(x_{1}\right)=3 w_{1}^{4}+2 w_{2}^{4}+w_{3}^{4}+w_{4}^{4}, \\
& p_{A}\left(x_{2}\right)=3 w_{1}^{4}+2 w_{2}^{4}+2 w_{3}^{4}+w_{4}^{4}, \\
& p_{A}\left(x_{3}\right)=3 w_{1}^{4}+3 w_{2}^{4}+2 w_{3}^{4}+w_{4}^{4} .
\end{aligned}
$$

Taking into account duplication, the tie among all three alternatives holds; hence

$$
\begin{aligned}
& x_{1} \sim_{A} x_{2} \quad \Leftrightarrow \quad w_{3}^{4}=0, \\
& x_{1} \sim_{A} x_{3} \quad \Leftrightarrow \quad w_{2}^{4}+w_{3}^{4}=0, \\
& x_{1} \sim_{A} x_{3} \quad \Leftrightarrow \quad w_{2}^{4}=0 .
\end{aligned}
$$

Then, $w_{2}^{4}=w_{3}^{4}=0$. Once proven that central weights are null (this fact will be taken into account in what follows), what remains is to show that lateral weights in each side of the triangle should the same at any level, i.e., there exists $\alpha \in[0,1]$ such that $w_{1}^{m}=\alpha$ and $w_{m}^{m}=1-\alpha$, for every $m \geq 2$. To do this, consider $\alpha=\frac{p}{q}$ with $p, q \in \mathbb{N}$ and $p<q$, expressed as an irreductible fraction, and any profile with $m$ voters and $q+1$ alternatives where the alternative $x_{1}$ is at least the best for one voter and the worst for another one, while $x_{2}$ occupies the position $p+1$ for all of them. A sketch of such ad hoc profile would be 


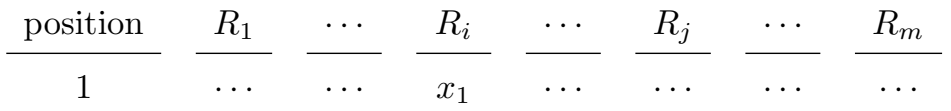

$$
\begin{aligned}
& \begin{array}{llllllll}
\ldots & \ldots & \ldots & \ldots & \ldots & \ldots & \ldots & \ldots
\end{array} \\
& \begin{array}{cccccccc}
p+1 & x_{2} & x_{2} & x_{2} & x_{2} & x_{2} & x_{2} & x_{2}
\end{array} \\
& \begin{array}{llllllll}
\cdots & \ldots & \ldots & \ldots & \ldots & \ldots & \ldots & \ldots
\end{array} \\
& \begin{array}{llllllll}
q+1 & \ldots & \ldots & \ldots & \ldots & x_{1} & \ldots & \ldots
\end{array}
\end{aligned}
$$

The aggregated positions for the selected alternatives would be

$$
\begin{aligned}
& p_{A}\left(x_{1}\right)=\frac{p}{q}(q+1)+\left(1-\frac{p}{q}\right)=p+1, \\
& p_{A}\left(x_{2}\right)=\frac{p}{q}(p+1)+\left(1-\frac{p}{q}\right)(p+1)=p+1,
\end{aligned}
$$

so that $x_{1} \sim_{A} x_{2}$, being $A$ the voting rule corresponding to any EOWA with such weights.

But now, if we replicate any subset of voters becoming the new weights $\beta \neq \alpha$ and hence $1-\beta \neq 1-\alpha$, then the new aggregated positions would be

$$
\begin{aligned}
& p_{A}\left(x_{1}\right)=\beta(q+1)+(1-\beta) \neq p+1, \\
& p_{A}\left(x_{2}\right)=\beta(p+1)+(1-\beta)(p+1)=p+1,
\end{aligned}
$$

so that $x_{1} \sim_{A} x_{2}$ does not hold. Hence, if lateral weights change from one dimension to another, duplication fails.

In conclusion, under duplication we obtain the class of weighting triangles

\begin{tabular}{ccccccccc} 
& & \multicolumn{1}{c}{1} & & & & \\
& & & & & & & \\
& & $\alpha$ & & $1-\alpha$ & & & \\
& & & & & & & \\
& $\alpha$ & & 0 & & $1-\alpha$ & & \\
& & & & & & & & \\
$\alpha$ & & 0 & & 0 & & $1-\alpha$ & \\
& & & & & & & \\
$\ldots$ & $\ldots$ & $\ldots$ & $\ldots$ & $\ldots$ & $\ldots$ & $\ldots$ & $\ldots$
\end{tabular}


As specific cases we have:

- $\alpha=1$ : maximum (maximin rule),

- $\alpha=0:$ minimum (maximax rule),

- $\alpha=0.5$ : mid-range.

It is worth mentioning that duplication is related to the Hurwicz criterion [28] used in decision making under complete uncertainty, where the value of a decision is a convex combination of its lowest possible expected value (pessimistic assessment) and of its highest one (optimistic assessment). On the other hand, although duplication might seem to be antidemocratic, Congar and Merlin [13] show that at least it is compatible with the basic democratic principle of anonymity and advocate it as a way "to protect the opinion of a minority against the will of the majority".

\subsection{An illustrative example}

Consider three voters that arrange three alternatives according to the following profile

\begin{tabular}{|c|c|c|}
\hline$R_{1}$ & $R_{2}$ & $R_{3}$ \\
\hline$x_{2}$ & $x_{2} x_{3}$ & $x_{1} x_{3}$ \\
\hline$x_{3}$ & $x_{1}$ & $x_{2}$ \\
\hline
\end{tabular}

with associated position matrix

$$
\left(\begin{array}{ccc}
3 & 1 & 2 \\
3 & 1.5 & 1.5 \\
1.5 & 3 & 1.5
\end{array}\right)
$$

If we choose the OWA operator $A_{\boldsymbol{w}(\alpha)}$ associated with the weighting vector $\boldsymbol{w}(\alpha)=(\alpha, 0,1-\alpha)$, with $\alpha \in[0,1]$, then the corresponding aggregated positions for the alternatives would be 


$$
\begin{aligned}
& p_{A_{\boldsymbol{w}(\alpha)}}\left(x_{1}\right)=3 \alpha+1.5(1-\alpha)=1.5 \alpha+1.5, \\
& p_{A_{\boldsymbol{w}(\alpha)}}\left(x_{2}\right)=3 \alpha+1(1-\alpha)=2 \alpha+1, \\
& p_{A_{\boldsymbol{w}(\alpha)}}\left(x_{3}\right)=2 \alpha+1.5(1-\alpha)=0.5 \alpha+1.5 .
\end{aligned}
$$

\begin{tabular}{|c|c|c|c|c|}
\hline$\alpha=0$ & $0<\alpha<\frac{1}{3}$ & $\alpha=\frac{1}{3}$ & $\frac{1}{3}<\alpha<1$ & $\alpha=1$ \\
\hline$x_{2}$ & $x_{2}$ & $x_{2} \quad x_{3}$ & $x_{3}$ & $x_{3}$ \\
\hline$x_{1} \quad x_{3}$ & $x_{3}$ & $x_{1}$ & $x_{2}$ & $x_{1} \quad x_{2}$ \\
\hline & $x_{1}$ & & $x_{1}$ & \\
\hline
\end{tabular}

According to the possible values of $\alpha$, the corresponding social orders are the following

As one could expect, different social orders appear depending on $\alpha$.

In the following subsection we propose an integrating method to obtain a unified result for each alternative taking into account the different outcomes when $\alpha$ ranges from 0 to 1 .

\subsection{Overall positions and social order}

For the general case with $n$ alternatives and using in a first stage the positional voting rule associated with the OWA operator of weighting vector $\boldsymbol{w}(\alpha)=(\alpha, 0, \ldots, 0,1-\alpha)$, it is possible to assign the corresponding social position $p_{A_{\boldsymbol{w}(\alpha)}}\left(x_{i}\right)$ to the alternative $x_{i}$. Thus, we can introduce the function $\mu_{i}:[0,1] \longrightarrow \mathbb{R}$ defined as $\mu_{i}(\alpha)=p_{A_{\boldsymbol{w}(\alpha)}}\left(x_{i}\right)$. Such function is always a degree one polynomial (a linear function), and hence Riemann integrable. This fact allows us to define the overall position of $x_{i}$ as

$$
p\left(x_{i}\right)=\int_{0}^{1} \mu_{i}(\alpha) d \alpha .
$$

Easy computations lead to the following results in the previous example: 


$$
\begin{aligned}
& p\left(x_{1}\right)=\int_{0}^{1} \mu_{1}(\alpha) d \alpha=\int_{0}^{1}(1.5 \alpha+1.5) d \alpha=9 / 4, \\
& p\left(x_{2}\right)=\int_{0}^{1} \mu_{2}(\alpha) d \alpha=\int_{0}^{1}(2 \alpha+1) d \alpha=2, \\
& p\left(x_{3}\right)=\int_{0}^{1} \mu_{3}(\alpha) d \alpha=\int_{0}^{1}(0.5 \alpha+1.5) d \alpha=7 / 4 .
\end{aligned}
$$

Thus, the overall social order is $x_{3} \succ x_{2} \succ x_{1}$.

In conclusion, for each $\alpha \in[0,1]$ the corresponding positional voting rule associated with $A_{\boldsymbol{w}(\alpha)}$ only takes into account the best and worst positions for each alternative, yielding different social orders in each case. However, the possible criticism on the influence of the choice of $\alpha$ in the result can be mitigated under this overall approach, where a social order is obtained not corresponding with any predetermined $\alpha$, but amalgamating all allowable values for this parameter.

\section{Concluding remarks}

In this paper we have presented a general framework for positional voting rules which includes all scoring rules as especial cases. To this aim, we need an aggregation process for obtaining a collective position from individual ones for each alternative. This is the reason why we have mainly used OWA operators, as they provide a comprehensive way to deal with this kind of information. More concretely, we have analyzed how the maximum and the minimum OWA operators induce the so called maximin and maximax voting rules, respectively, recently characterized by Congar and Merlin [13]. Of course, these rules are not scoring rules (they satisfy duplication, a property radically opposed to the continuity verified by the scoring rules) although all of them share interesting features due to their positional nature. A comprehensive diagram showing our knowledge of the logical relationship among these rules appears in Figure 3.

We have focused on the duplication property appearing in the above mentioned characterization. On our part, once introduced suitable extended notions 


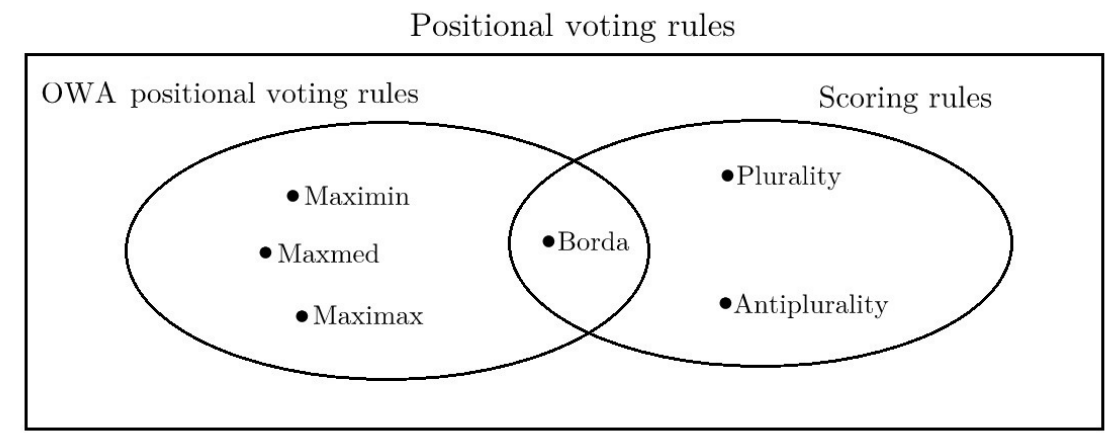

Figure 3: Relationship among positional voting rules

to take into account a variable electorate, we have characterized all EOWAgenerated positional voting rules satisfying that property.

Some questions remain unanswered. As proven in Prop. 1, all scoring rules are positional voting rules. Even more, some scoring rules are positional voting rules generated by OWA operators. This is the case of the Borda rule, associated with the arithmetic mean. However, a characterization of the family of scoring rules that are generated by OWA operators (in other words, the relationship among scores and weights) is to be found. Additionally, families of aggregation functions other than OWA operators, such as quasiarithmetic means, could be also taken into account.

\section{Acknowledgments}

The authors would like to thank Jorge Alcalde-Unzu, Eyal Baharad, JeanFrançois Laslier, Vincent Merlin, Shmuel Nitzan, Marina Núñez, Arunava Sen and Arkadii Slinko for their comments.

We are grateful for the financial support of the Spanish Ministerio de Economía y Competitividad (project ECO2012-32178) and Consejería de Educación de la Junta de Castilla y León (project VA066U13). 


\section{References}

\section{References}

[1] Baharad E, Nitzan S. Ameliorating majority decisiveness through expression of preference intensity. American Political Science Review 2002;96:745754 .

[2] Baharad E, Nitzan S. The inverse plurality rule - an axiomatization. Social Choice and Welfare 2005;25:173-178.

[3] Balinski M, Laraki R. A theory of measuring, electing and ranking. Proceedings of the National Academy of Sciences of the United States of America (PNAS) 2007;104:8720-8725.

[4] Balinski M, Laraki R. Majority Judgment: Measuring Ranking and Electing. MIT Press, Cambridge MA; 2011.

[5] Bassett GW, Persky J. Robust voting. Public Choice 1999;99:299-310.

[6] Beliakov G, Bustince Sola H, Calvo Sánchez T. A Practical Guide to Averaging Functions, Springer, Heidelberg; 2016.

[7] Beliakov G, Pradera A, Calvo T. Aggregation Functions: A Guide for Practitioners, Springer, Heidelberg; 2007.

[8] Black D. Partial justification of the Borda count. Public Choice 1976;28:115.

[9] Brams SJ, Kilgour DM. Fallback bargaining. Group Decision and Negotiation 2001;10:287-316.

[10] Calvo T, Kolesárova A, Komorníková M, Mesiar R. Aggregation operators: Properties, classes and construction methods, in: T Calvo, G Mayor, R Mesiar (Eds.), Aggregation Operators: New Trends and Applications, Physica-Verlag, Heidelberg, pp 3-104; 2002. 
[11] Calvo T, Mayor G. Remarks on two types of extended aggregation functions. Tatra Mountains Mathematical Publications 1999;16:235-253.

[12] Chebotarev PY, Shamis E. Characterizations of scoring methods for preference aggregation. Annals of Operations Research 1998;80:299-332.

[13] Congar R, Merlin V. A characterization of the maximin rule in the context of voting. Theory and Decision 2012;72:131-147.

[14] Cook WD, Seiford LM. On the Borda-Kendall consensus method for priority ranking problems. Management Science 1982;28:621-637.

[15] Courtin S, Mbih B, Moyouwou I. Are Condorcet procedures so bad according to the reinforcement axiom? Social Choice and Welfare 2014;42:927940.

[16] Fagin R, Wimmners EL. A formula for incorporating weights into scoring rules. Theoretical Computer Science 2000;239:309-338.

[17] Falcó E, García-Lapresta JL. A distance-based extension of the Majority Judgement voting system". Acta Universitatis Matthiae Belii, series Mathematics 2011;18:17-27.

[18] Felsenthal DS. Review of paradoxes afflicting procedures for electing a single candidate, in: DS Felsenthal, M Machover (Eds.), Electoral Systems: Paradoxes, Assumptions, and Procedures, Springer-Verlag, Berlin, 19-91; 2012.

[19] Fodor J, Marichal JL, Roubens M. Characterization of the ordered weighted averaging operators. IEEE Transtactions on Fuzzy Systems 1995;3:236-240.

[20] García-Lapresta JL, Llamazares B, Peña T. Scoring rules and consensus, in: S Greco, R Marques Pereira, M Squillante, RR Yager, J Kacprzyk (Eds.), Preferences and Decisions: Models and Applications, SpringerVerlag, Berlin, 211-224; 2010. 
[21] García-Lapresta JL, Llamazares B, Peña T. Generating OWA weights from individual assessments, in: RR Yager, J Kacprzyk, G Beliakov, (Eds.), Recent Developments in the OWA Operators, Springer-Verlag, Berlin, 135$147 ; 2011$.

[22] García-Lapresta JL, Marley AAJ, Martínez-Panero M. Characterizing bestworst voting systems in the scoring context. Social Choice and Welfare 2010;34:487-496.

[23] García-Lapresta JL, Martínez-Panero M. Linguistic-based voting through centered OWA operators. Fuzzy Optimization and Decision Making 2009;8:381-393.

[24] García-Lapresta JL, Martínez-Panero M. Duplication in OWA-generated positional aggregation rules, in: H Bustince, J Fernández, R Mesiar, T Calvo, (Eds.), Aggregation Functions in Theory and Practise. Proceedings of the 7th International Summer School on Aggregation Operators, Springer-Verlag, Berlin, 117-128; 2013.

[25] García-Lapresta JL, Pérez-Román D. Measuring consensus in weak orders, in: E Herrera-Viedma, JL García-Lapresta, J Kacprzyk, H Nurmi, M Fedrizzi, S Zadrożny, (Eds.), Consensual Processes, STUDFUZZ, vol. 267. Springer-Verlag, Berlin, 213-234; 2011.

[26] Gärdenfors P. Positionalist voting functions. Theory and Decision 1973;4:124.

[27] Grabisch M, Marichal JL, Mesiar R, Pap E. Aggregation Functions. Cambridge University Press, Cambridge; 2009.

[28] Hurwicz L. A class of criteria for decision-making under ignorance. Cowles Commission Discussion Paper: Statistics 356; 1951.

[29] Hurwicz L, Sertel MR. Designing mechanisms, in particular for electoral systems: the majoritarian compromise. In: MR Sertel (Ed.): Economic Design and Behaviour. Macmillan, London; 1999. 
[30] Kacprzyk J, Nurmi H, Zadrożny S. The role of the OWA operators as a unification tool for the representation of collective choice sets, in: RR Yager, J Kacprzyk, G Beliakov, (Eds.), Recent Developments in the OWA Operators, Springer-Verlag, Berlin, 149-166; 2011.

[31] Laslier JF. Rank-based choice correspondences. Economics Letters $1996 ; 52: 279-286$.

[32] Laslier JF. And the loser is ... plurality voting, in: DS Felsenthal, M Machover (Eds.), Electoral Systems: Paradoxes, Assumptions, and Procedures, Springer-Verlag, Berlin, 327-351; 2012.

[33] Laslier JF. On choosing the alternative with the best median evaluation. Public Choice 2012;153:269-277.

[34] Levin J, Nalebuff B. An introduction to vote-counting schemes. Journal of Economic Perspectives 1995;9:3-26.

[35] Llamazares B. Choosing OWA operator weights in the field of Social Choice. Information Sciences 2007;177:4745-4756.

[36] Llamazares B, Peña T. Positional voting systems generated by cumulative standings functions. Group Decision and Negociation 2015;24:777-801.

[37] Llamazares B, Peña T. Scoring rules and social choice properties: some characterizations. Theory and Decision 2015;78:429-450.

[38] Mayor G, Calvo T. On extended aggregation functions. Proceedings of IFSA 97, vol. I, Prague, 281-285; 1997.

[39] Pattanaik PK. Positional rules of collective decision-making, in KJ Arrow, AK Sen, K Suzumura (Eds.), Handbook of Social Choice and Welfare, Volume 1, Elsevier, Amsterdam, 361-394; 2002.

[40] Rawls J. Distributive justice, in: E Phelps (Ed.), Economic Justice: Selected Readings, Penguin Education, Harmondsworth, 319-362; 1973. 
[41] Riker WH. Liberalism versus Populism. W.H. Freeman, San Francisco; 1982.

[42] Smith JH. Aggregation of preferences with variable electorate. Econometrica 1973;41:1027-1041.

[43] Smith WD. Range Voting website, in http://rangevoting.org/.

[44] Tideman N. Collective Decisions and Voting: The Potential for Public Choice. Ashgate Pubishing, Burlington; 2006.

[45] Wagman L, Conitzer V. False-name-proof voting with costs over two alternatives. International Journal of Game Theory 2014;43:599-618.

[46] Wang YM, Luo Y, Hua Z. Aggregating preference rankings using OWA operator weights. Information Sciences 2007;177:3356-3363.

[47] Yager RR. On ordered weighted averaging operators in multicriteria decision making. IEEE Transactions on Systems Man and Cybernetics 1988;8:183-190.

[48] Yager RR. Centered OWA operators. Soft Computing 2007;11:631-639.

[49] Yager RR, Kacprzyk J. (Eds.) The Ordered Weighted Averaging Operators: Theory and Applications. Kluwer Academic Publishers, Boston; 1997.

[50] Yager RR, Kacprzyk J, Beliakov G. (Eds.) Recent Developments in the Ordered Weighted Averaging Operators: Theory and Practice. SpringerVerlag, Berlin; 2011.

[51] Yokoo M, Sakurai Y, Matsubara B. The effect of false-name bids in combinatorial auctions: New frauds in internet auctions. Games and Economic Behavior 2005;46:174-188.

[52] Young HP. An axiomatization of Borda's rule. Journal of Economic Theory 1974;9:43-52. 
[53] Young HP. Social choice scoring functions. SIAM Journal on Applied Mathematics 1975;28:824-838.

[54] Xia L, Conitzer V. Generalized scoring rules and the frequency of coalitional manipulability. Proceedings of the 9th ACM Conference on Electronic Commerce, 109-118; 2008.

[55] Xia L, Conitzer V. Finite local consistency characterizes generalized scoring rules. Proceedings of the Twenty-First International Joint Conference on Artificial Intelligence, 336-341; 2009. 